\title{
Síntese e avaliação da atividade antileishmanial in vitro de bioisósteros do resveratrol ${ }^{1}$
}

\author{
Synthesis and in vitro evaluation of the antileishmanial \\ activity of resveratrol bioisosteres
}

\author{
Natália Prado da Silva ${ }^{2}$ \\ Luciana Maria Ribeiro Antinarelli ${ }^{3}$ \\ Juliana Alves dos Santos ${ }^{4}$ \\ Adilson David da Silva ${ }^{3}$ \\ Raíssa Soares Meinel ${ }^{3}$ \\ Ayla das Chagas Almeida ${ }^{5}$ \\ Rodrigo Pedro Pinto Soares ${ }^{6}$ \\ Elaine Soares Coimbra ${ }^{7}$
}

DOI: https://doi.org/10.34019/2179-3700.2018.v18.29863

\section{Resumo}

As leishmanioses são infecções causadas por protozoários do gênero Leishmania, responsáveis por altas taxas de morbidade e mortalidade em todo mundo. O tratamento está restrito a um número limitado de fármacos com alta toxicidade, variabilidade na eficácia e alto custo. Neste trabalho foram sintetizados quatro bioisósteros do resveratrol que foram avaliados em formas promastigotas de Leishmania amazonensis e $L$. braziliensis associadas à manifestação tegumentar da doença, além da citotoxicidade em macrófagos murinos. Dois dos compostos, 1a e 1b exibiram promissores resultados, com $\mathrm{Cl}_{50}$ variando de 3,0 a $16,44 \mu \mathrm{M}$, e não apresentaram toxicidade em macrófagos peritoneais. O composto $1 \mathrm{~b}$ exibiu o maior índice de seletividade $(>25)$ para ambas as espécies. Esses resultados indicam o potencial desta classe de compostos para o desenvolvimento de novos agentes antileishmaniais e estimulam a realização de estudos em amastigotas intracelulares e a avaliação do seu mecanismo de ação.

Palavras-chaves:Leishmania sp. Bioisósteros. Resveratrol. Tratamento.

\section{Abstract}

The leishmaniases are infections caused by protozoa of the Leishmania genus, accountable for

\footnotetext{
${ }^{1}$ Trabalho premiado no Seminário de Iniciação Científica da UFJF de 2014.

2 Bolsista do Programa PIBIC/CNPq/UFJF

${ }^{3}$ Pós-doutoranda CNPq/UFMG

${ }^{4}$ Departamento de Química/ICE/UFJF

${ }^{5}$ Doutoranda PPgCbio/UFJF

${ }^{6}$ Centro de Pesquisas René Rachou/Fiocruz-BH

${ }^{7}$ Professora Orientadora. Departamento de Parasitologia, Microbiologia e Imunologia/ICB/UFJF. elaine.coimbra@ufjf.edu.br
} 
high levels of morbidity and mortality worldwide. The treatment is restricted to few drugs with high toxicity, variability in efficacy and high cost. In this work, four resveratrol bioisosteres were synthesized and evaluated in promastigotes of Leishmaniaamazonensis and L. braziliensis, associated with the cutaneous form of the disease, and were also assayed for their cytotoxicity in murine macrophages. The compounds $1 \mathrm{a}$ and $\mathbf{1 b}$ showed promising results, with $\mathrm{IC}_{50}$ ranging from 3.0 to $16.44 \mu \mathrm{M}$, and showing no toxicity in peritoneal macrophages. Compound $\mathbf{1 b}$ exhibited the highest selectivity index (> 25) for both Leishmania species. These results indicate the potential of this class of compounds in the development of new antileishmanial agents and stimulate the study of intracellular amastigotes and the evaluation of their mechanism of action.

Keywords: Leishmaniasp. Bioisosteres. Resveratrol. Treatment.

\section{INTRODUÇÃO}

As leishmanioses são infecções causadas por protozoários do gênero Leishmania, responsáveis por altas taxas de morbidade e mortalidade em todo mundo. Mais de 20 espécies de Leishmania podem infectar o homem e juntamente com a resposta imunológica do hospedeiro podem levar a diferentes manifestações clínicas, classicamente divididas em cutânea e visceral (AMEEN, 2010). A leishmaniose cutânea é a forma mais comum da doença, com 1,5 milhões de novos casos por ano (WHO, 2012). Apesar desta forma clínica não estar associada à mortalidade, pode gerar desfigurações graves, ocasionando grande estigma social (AMEEN, 2010).

Não existe vacina disponível para estas doenças e o tratamento está restrito a um número limitado de fármacos. Os antimoniais pentavalentes são considerados como primeira linha de tratamento e anfotericina $B$, pentamidina, miltefosina e paramomicina são considerados como de segunda linha (ULIANA et al., 2018). No entanto, todos esses medicamentos apresentam diversas limitações devido aos graves efeitos adversos, variabilidade na eficácia e longa duração do tratamento (DE MENEZES et al., 2015). Nesse sentido, o desenvolvimento de novas opções terapêuticas é necessário.

O resveratrol $(3,5,4$ '-trihidroxiestilbeno) é um composto polifenólico produzido por variadas espécies de plantas e alimentos, como uva e amendoim (KOUSHKI et al., 2018) e algumas atividades biológicas podem ser reportadas, tais como efeitos antioxidantes (XIA et al., 2017), antiinflamatórios (TUNG et al., 2015) e anticancerígenos (PAREKH et al., 2011). A atividade antileishmanial do resveratrol e de alguns análogos também já foi relatada (FERRREIRA et al., 2014; PASSOS et al., 2015). Recentemente, nosso grupo de pesquisa reportou a atividade antileishmanial de alguns análogos do resveratrol (DE PAULA et al., 2013; COIMBRA et al., 2016), além da possível ação sobre a enzima tripanotiona redutase (DA SILVA et al., 2018), essencial componente de defesa antioxidante do parasito (FAIRLAMB et al., 1985). 
O termo bioisosterismo foi introduzido por Friedman, em 1951, como um fenômeno observado entre substâncias estruturalmente relacionadas que apresentam propriedades biológicas similares ou antagônicas (FRIEDMAN, 1951). Trata-se de uma estratégia da Química Medicinal utilizada para modificação estrutural de moléculas com atividade biológica (LIMA, 2005). Além da potencialização da atividade farmacológica dos compostos, outra vantagem do uso dessa estratégia é o aumento de seletividade com simultânea diminuição de efeitos adversos (LIMA, 2005).

Neste trabalho foi avaliada a atividade de bioisósteros do resveratrol em diferentes espécies de Leishmania, bem comoa citotoxicidade em macrófagos murinos.

\section{METODOLOGIA}

\subsection{Compostos químicos}

Os compostos mostrados no Esquema 1 foram obtidos por condensação de 2aminofenol com quantidade equimolar de 3-hidróxi-4-metóxibenzaldeído e ácido 4formilbenzóico em etanol. Os dados espectroscópicos do composto 1a foram condizentes com a literatura (BAE et al., 2012). Por outro lado, o composto 1b é inédito e os dados estão descritos abaixo.

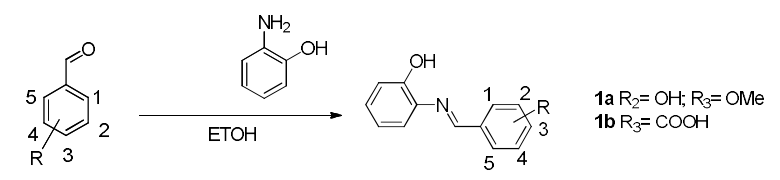

Esquema 1. Derivados obtidos a partir do 2-aminofenol.

Os compostos mostrados no Esquema 2 foram obtidos por condensação de N-(7cloroquinolin-4-il)-etanodiamina (1 mmol) com 3-hidróxi-4-metoxibenzaldeído e ácido 4formilbenzóico $(1,2 \mathrm{mmol})$ utilizando etanol como solvente. Os mesmos foram obtidos como sólidos, após serem filtrados, lavados com etanol e secos em estufa.

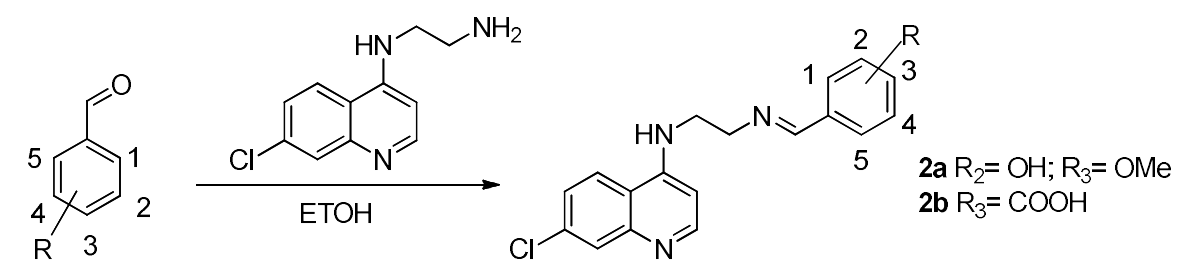

Esquema 2. Derivados obtidos a partir da N-(7-cloroquinolin-4-il)-etanodiamina.

\subsubsection{Sínteses}

Ácido (E)-4-(((2-hidróxifenil)imino)-metil)-benzoico (1b). A um balão de fundo redondo 
adicionou-se 0,109 g (1 mmol) de 2-aminofenol e solubilizou-se em $5 \mathrm{~mL}$ de etanol. Foram adicionados $0,150 \mathrm{~g}(1 \mathrm{mmol})$ de ácido 4 -formilbenzóico ao meio reacional. A reação foi mantida à temperatura ambiente durante 24 horas até a formação de um precipitado amarelo, que foi filtrado e lavado com etanol. $(0.178 \mathrm{~g}, 74 \%) .{ }^{1} \mathrm{H}$ NMR $(500 \mathrm{MHz}$, DMSOd6) $\delta{ }^{1} \mathrm{H}$ NMR (500 MHz, DMSO-d6) $\delta 9.13(\mathrm{~s}, 1 \mathrm{H}), 8.81(\mathrm{~s}, 1 \mathrm{H}), 8.14(\mathrm{~d}, J=8.4 \mathrm{~Hz}, 2 \mathrm{H})$, $8.06(\mathrm{~d}, J=8.2 \mathrm{~Hz}, 2 \mathrm{H}), 7.26(\mathrm{dd}, J=7.9,1.5 \mathrm{~Hz}, 1 \mathrm{H}), 7.12(\mathrm{t}, J=7.7 \mathrm{~Hz}, 1 \mathrm{H}), 6.92(\mathrm{dd}, J$ $=8.1,1.0 \mathrm{~Hz}, 1 \mathrm{H}), 6.86(\mathrm{t}, J=7.6 \mathrm{~Hz}, 1 \mathrm{H}) .{ }^{13} \mathrm{C} \mathrm{NMR}(126 \mathrm{MHz}$, DMSO) $\delta 167.4,158.7$, $151.9,140.5,137.9,133.2,130.0,129.3,128.4,119.9,119.8,116.7$.

(E)-5-(((2-((7-cloroquinolin-4-il)-amino)-etil)-imino)-metil)-2-metóxifenol (2a). 3-hidróxi4-metóxibenzaldeído, 0,182 g (1,2 mmol) foi dissolvido em etanol em um béquer $(5 \mathrm{~mL}) \mathrm{e}$ posteriormente, $0,221 \mathrm{~g}(1,0 \mathrm{mmol})$ de $\mathrm{N}$-(7-cloroquinolin-4-il)-etanodiamina foi adicionado a esta solução e deixado em agitação constante à temperatura ambiente. A mistura reacional foi monitorada por cromatografia em camada fina durante 24 horas. Após este período, observou-se a formação de um precipitado amarelo na solução que foi filtrado e lavado com etanol. (0.248 g, 70\%). RMN de ${ }^{1} \mathrm{H}(500 \mathrm{MHz}$, DMSO-d6) $\delta(\mathrm{ppm}), J(\mathrm{~Hz}): 8.40$ (d, $J=5.5$ ), 8.24 (d, $J=9.0$ ), 8.17 (s), 7.78 (d, $J=2.5$ ), 7.43 (dd, $J=2.0$ e 9.0), 7.23 (d, $J$ $=1.5$ ), 7.06 (dd, $J=2.0$ e 8.5), 6.94 (d, $J=8.0), 6.59$ (d, $J=5.5), 3.81(\mathrm{~m}), 3.574(\mathrm{~m})$. $\mathrm{RMN}{ }^{13} \mathrm{C}(126 \mathrm{MHz}$, DMSO-d6), $\delta$ (ppm):162.2, 152.3, 150.5, 149.9, 147.0, 133.8, 129.6, 127.9, 124.4, 121.4, 117.9, 113.8, 111.9, 99.5, 59.0, 56.0, 43.7.

Ácido (E)-4-(((2 -((7-cloroquinolin-4-il)-amino)-etil)-imino)-metil)-benzoico (2b).Ácido 4-formilbenzóico $0,180 \mathrm{~g}(1,2 \mathrm{mmol})$ foi dissolvido em etanol $(5 \mathrm{~mL})$ em um balão, posteriormente, $0,221 \mathrm{~g}(1,0 \mathrm{mmol})$ de $\mathrm{N}$-(7-cloroquinolin-4-il)-etanodiamina foi adicionado a esta solução e deixada em agitação à temperatura ambiente. A mistura reacional foi monitorada por cromatografia em camada fina durante 24 horas. Após este período, observou-se a formação de um precipitado amarelo, que foi filtrado e lavado com etanol. (0.290 g, 82\%). RMN de ${ }^{1} \mathrm{H}(500 \mathrm{MHz}$, DMSO-d6) $\delta 8.40$ (d, 1H, J = 5.5 Hz), 8.27 (d, $1 \mathrm{H}, J$ $=9.0 \mathrm{~Hz}$ ), $8.19(\mathrm{~s}, 1 \mathrm{H}), 7.79(\mathrm{~d}, 1 \mathrm{H}, J=2.0 \mathrm{~Hz}), 7.55(\mathrm{~d}, 2 \mathrm{H}, J=8.5 \mathrm{~Hz}), 7.43(\mathrm{dd}, 1 \mathrm{H}, J=$ $2.0 \mathrm{~Hz}$ and $9.0 \mathrm{~Hz}$ ), $682(\mathrm{~d}, 2 \mathrm{H}, J=8.5 \mathrm{~Hz}), 6.59(\mathrm{~d}, 1 \mathrm{H}, J=5.5 \mathrm{~Hz}), 3.80(\mathrm{t}, 4 \mathrm{H}, J=6.0$ Hz). RMN ${ }^{13} \mathrm{C}(126 \mathrm{MHz}$, DMSO-d6) $\delta 167.7,162.2,151.9,150.8,149.0,139.7,134.1$, $133.9,130.0,128.3,124.7,124.5,117.7,99.5,59.3,43.5$. 


\subsection{Atividade biológica}

\subsubsection{Cultivo dos parasitos}

Promastigotas de $L$. amazonesis (IFLA/BR/67/PH8) e $L$. braziliensis (MHOM/Br/75/M2903) foram cultivadas a $25^{\circ} \mathrm{C}$, em meio Warren (preparado com meio $\mathrm{BHI}$ suplementado com ácido fólico e hemina) e meio BHI (Infusão de cérebro e coração bovino), respectivamente. Os meios foram suplementados com 10\% de Soro Bovino Fetal (SBF) (Cultilab, São Paulo, Brasil) e antibióticos.

\subsubsection{Ensaio antipromastigota}

Promastigotas de L. amazonensis e L. brazilensis, emfase exponencial de crescimento, foram adicionadas em placas de 96 poços, juntamente com os compostos $(3,12$ a $100 \mu \mathrm{M})$, por $72 \mathrm{~h}$. A inibição do crescimento das formas promastigotas foi determinada pelo teste colorimétrico do MTT (3-(4,5-dimetiltiazol-2-il)-2-5 difenil tetrazólico-Sigma Aldrich- St. Louis, MO, USA), considerando os parasitos sem adição dos compostos como controle negativo (100\% de viabilidade). Os resultados foram expressos como concentração molar que inibe $50 \%$ do crescimento parasitário $\left(\mathrm{Cl}_{50}\right)$. Os ensaios foram realizados em duplicata em três experimentos independentes.

\subsubsection{Ensaio de citotoxicidade em macrófagos}

Macrófagos peritoneais foram coletados de camundongos BALB/c, previamente inoculados com tioglicolato de sódio a $3 \%$. As células $\left(2 \times 10^{6}\right.$ células $\left./ \mathrm{mL}\right)$ foram adicionadas em placas de 96 poços em meio RPMI suplementado com $10 \%$ de SBF, a $33^{\circ} \mathrm{C}$ com $5 \% \mathrm{CO}_{2}$ por $24 \mathrm{~h}$. Após lavagem com PBS estéril, células aderentes foram incubadas com os compostos em diferentes concentrações (9,37 a $150 \mu \mathrm{M})$ por 72 h. A viabilidade dos macrófagos foi determinada pelo método do MTT (MOSSMAN, 1983). Os ensaios foram feitos em duplicata em três experimentos independentes. Os procedimentos foram realizados de acordo com os protocolos aprovados pelo Comitê de Ética em Pesquisa Animal da Universidade Federal de Juiz de Fora (\#CEUA 013/2015). O índice de seletividade dos compostos foi determinado pela razão entre o $\mathrm{CC}_{50}$ sobre macrófagos e o $\mathrm{Cl}_{50}$ sobre o parasito na forma promastigota. 


\subsubsection{Análise estatística}

$\mathrm{O} \mathrm{Cl}_{50}$ e $\mathrm{CC}_{50}$ foram determinados pelo programa Grafit versão 5.

\section{RESULTADOS E DISCUSSÃO}

O presente estudo investigou o efeito antileishmanial de quatro compostos sintéticos bioisósteros do resveratrol, $\mathbf{1 a}, \mathbf{1} \mathbf{b}, \mathbf{2 a}$ e $\mathbf{2 b}$ (Tabela 1). A atividade antileishmanial dos bioisósteros foi avaliada em formas promastigotas de L. amazonensis e L. braziliensis, espécies associadas a manifestações tegumentares da doença (SILVEIRA et al., 2009). A citotoxicidade dos compostos em células de mamíferos foi também determinada e, para tal, utilizou-se macrófagos peritoneais de camundongos BALB/c. Como pode ser verificado na Tabela 1, os compostos 1a e $\mathbf{1 b}$ apresentaram expressiva atividade em ambas as espécies de Leishmania avaliadas. O composto 1a com $\mathrm{Cl}_{50}$ de 11,03 e $16,44 \mu \mathrm{M}$ e o composto 1 b com $\mathrm{Cl}_{50}$ de 4,36 e 5,82 $\mu \mathrm{M}$ para $L$. brazilensis e L. amazonensis, respectivamente. Os mesmos foram mais efetivos do que o fármaco de referência, miltefosina. Por outro lado, os compostos $\mathbf{2 a}$ e $\mathbf{2 b}$ não mostraram atividade em formas promastigotas de Leishmania nas concentrações avaliadas.

Em um trabalho recente publicado por nosso grupo de pesquisa (DA SILVA et al., 2018), o efeito antileishmanial do resveratrol foi avaliado e o $\mathrm{Cl}_{50}$ obtido para a substância em formas promastigotas de $L$. braziliensis e $L$. amazonensis foi de 75,13 e 22,23 $\mu \mathrm{M}$, respectivamente. De modo interessante, os bioisósteros $\mathbf{1 a}$ e $\mathbf{1 b}$ foram mais ativos do que o resveratrol quando testados nas mesmas espécies de Leishmania.

Tabela 1- Avaliação da atividade antileishmanial, citotoxicidade em macrófagos murinos e índice de seletividade dos bioisósteros do resveratrol.

\begin{tabular}{|c|c|c|c|c|c|}
\hline \multirow[t]{2}{*}{ Compostos } & \multicolumn{2}{|c|}{$\begin{array}{r}\text { Promastigotas } \\
\mathrm{Cl}_{50}{ }^{a}(\mu \mathrm{M})\end{array}$} & \multicolumn{2}{|c|}{ IS } & \multirow[t]{2}{*}{$\begin{array}{l}\text { Macrófagos } \\
\mathrm{CC}_{50}{ }^{\mathrm{b}}(\mu \mathrm{M})\end{array}$} \\
\hline & L. $a m a^{*}$ & L. braz** & L. $a m a^{*}$ & L. braz** & \\
\hline $1 a$ & $16,44 \pm 0,41$ & $11,03 \pm 1,03$ & $>9,1$ & $>13,6$ & $>150,0$ \\
\hline $1 \mathrm{~b}$ & $5,82 \pm 0,92$ & $4,36 \pm 0,55$ & $>25,8$ & $>34,4$ & $>150,0$ \\
\hline $2 a$ & $>100,0$ & $>100,0$ & & 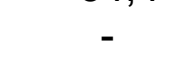 & $>150,0$ \\
\hline $2 b$ & $>100,0$ & $>100,0$ & - & - & $102,4 \pm 11,6$ \\
\hline Resveratr & $22,23 \pm 2,30$ & $75,13 \pm 7,60$ & 5,4 & 1,6 & $120,22 \pm 35,71$ \\
\hline Miltefosina & $22,0 \pm 0,5$ & $29,66 \pm 0,02$ & 6,0 & 4,4 & $131,9 \pm 3,9$ \\
\hline
\end{tabular}

Fonte: elaboração própria.

${ }^{\mathrm{a}} \mathrm{Cl}_{50}$ : Concentração que inibe $50 \%$ do crescimento dos parasitos. ${ }^{\mathrm{b}} \mathrm{CC}_{50}$ : Concentração que inibe $50 \%$ da viabilidade dos macrófagos. IS= Índice de Seletividade. ${ }^{*} \mathrm{~L}$. ama(L. amazonensis) ${ }^{* *} \mathrm{~L}$. braz ( $L$. braziliensis).Miltefosina foi utilizada como droga controle (controle positivo). 
Numa análise comparativa do efeito dos compostos nas diferentes espécies de Leishmania avaliadas, observou-se um melhor perfil de atividade dos compostos para a espécie $L$. braziliensis. É bem documentado na literatura que as diferenças bioquímicas e moleculares entre as espécies de Leishmania resultam em variações na sensibilidade à fármacos. A miltefosina, por exemplo, apresenta eficácia in vitro altamente heterogênea entre espécies de Leishmania do Novo Mundo e do Velho Mundo (YARDLEY et al., 2005; MORAIS-TEIXEIRA et al., 2011). A variação na sensibilidade de diferentes drogas utilizadas no tratamento das leishmanioses já foi demonstrada até mesmo entre isolados clínicos distintos de L. braziliensis (RUGANI et al., 2018). Portanto, não é surpreendente a variação na sensibilidade observada entre Leishmania spverificada no presente trabalho.

$\mathrm{Na}$ triagem por novos compostos com ação antileishmanial a avaliação do perfil de citotoxicidade em macrófagos, principal célula hospedeira da Leishmania, é um parâmetro fundamental a ser avaliado. A atividade citotóxica dos compostos foi avaliada em macrófagos peritoneais de camundongos e os resultados foram apresentados na Tabela 1. É interessante verificar que os bioisósteros que apresentaram atividade antileishmanial (1a e 1b) não apresentaram toxidez para macrófagos, mesmo em concentrações mais elevadas das substâncias $(150 \mu \mathrm{M})$. No trabalho realizado pelo nosso grupo, o $\mathrm{CC}_{50}$ obtido para o resveratrol foi de 120,22 $\mu \mathrm{M}$ (DA SILVA et al., 2018).

Outro parâmetro importante a ser avaliado na triagem in vitro de novas moléculas antileishmaniais é o índice de seletividade (IS), que expressa a relação entre a citotoxicidade da droga em células de mamíferos com sua atividade farmacológica. A literatura recomenda que o valor de IS satisfatório para moléculas com potencial antileishmanial deve ser superior a 10.0 (LAVORATO et al., 2017). Os compostos 1a e 2a apresentaram valores de IS próximos ou superiores a 10, demonstrando a ação seletiva destes compostos para o parasito, sem causar toxicidade para a célula hospedeira. Miltefosina e resveratrol exibiram valores de IS $<10$ para ambas as espécies avaliadas. Um valor satisfatório IS é altamente desejável para predizer a segurança in vivo da molécula sobre as células do hospedeiro humano. Em se tratando do desenvolvimento de novas drogas para a quimioterapia das leishmanioses, este é um ponto-chave, visto que a principal limitação dos fármacos atualmente utilizados no tratamento da doença são os graves efeitos adversos relacionados à sua elevada toxicidade renal, hepática e cardíaca (DE MENEZES et al., 2015). 


\section{CONCLUSÕES}

Neste trabalho, quatro bioisósteros do resveratrol foram sintetizados e sua atividade antileishmanial in vitro e citotoxicidade em macrófagos murinos foi avaliada. Os compostos 1a e 1b exibiram atividade antileishmanial promissora e seletiva em $L$. amazonensis e L. braziliensis, importantes espécies associadas às manifestações cutâneas da doença. Os resultados indicam o potencial desta classe de compostos para o desenvolvimento de novos agentes antileishmaniais e estimulam a continuidade dos estudos.

\section{AGRADECIMENTOS}

Os autores agradecem ao Conselho Nacional de Desenvolvimento Científico e Tecnológico (CNPq), Fundação de Amparo à Pesquisa do Estado de Minas Gerais (FAPEMIG), Coordenação de Aperfeiçoamento de Pessoal de Ensino Superior (CAPES) e Universidade Federal de Juiz de Fora (UFJF) pelo apoio com bolsa e/ou suporte financeiro.

\section{REFERÊNCIAS}

AMEEN, M. Cutaneous leishmaniasis: advances in disease pathogenesis, diagnostics and therapeutics. Clinical and Experimental Dermatology: Clinical Dermatology, Portsmouth, v. 35, n. 7, p. 699-705, 2010.

BAE, S.J.H., et al.. Design, synthesis, and evaluation of (E)-N-substituted benzylideneaniline derivatives as tyrosinase inhibitors. European Journal Medicinal Chemistry, v. 57, p. 383-390, 2012.

COIMBRA, E. S. et al. Synthesis, antitubercular and leishmanicidal evaluation of resveratrol analogues. Journal of the Brazilian Chemical Society, Campinas, v. 27, n. 12, p. 2161-2169, 2016.

DA SILVA, A. D. et al. Insights about resveratrol analogues against trypanothione reductase of Leishmaniabraziliensis: molecular modeling, computational docking and in vitro antileishmanial studies. Journal of Biomolecular Structure and Dynamics, p. 1-34, 2018.

DE MENEZES, J. P. B. et al. Advances in development of new treatment for leishmaniasis. BioMed Research International, v. 2015, 2015.

DE PAULA, D. T. S et al. Synthesis, cytotoxicity and antileishmanial activity of aza-stilbene derivatives. Mediterranean Journal of Chemistry, Kenitra, v. 2, n. 3, p. 493-502, 2013. 
FAIRLAMB, A. H. et al. Trypanothione: a novel bis (glutathionyl) spermidine cofactor for glutathione reductase in trypanosomatids. Science,Washington,v. 227, n. 4693, p. 14851487, 1985.

FERREIRA, C. et al. Resveratrol is active against Leishmaniaamazonensis: in vitro effect of its association with amphotericin B. Antimicrobial Agents and Chemotherapy, Washington, v. 58, n.10, p. 6197-6298, 2014.

FRIEDMAN, H. L. Influence of Isosteric Replacements upon Biological Activity. National Academy of Science, Washington, 1951, n. 206, p. 295.

KOUSHKI, M. et al. Resveratrol: A miraculous natural compound for diseases treatment. Food Science \& Nutrition, p. 1-18, 2018.

LAVORATO, S. N. et al. 1,3-Bis(aryloxy)propan-2-ols as potential antileishmanial agents.Chemical Biology and Drug Design, Oxford, v. 90, n. 15, p. 981-986, 2017.

LIMA, L.M., BARREIRO, E.J. Bioisosterism: a useful strategy for molecular modification and drug design. Current Medicinal Chemistry,Sharjah, v.12, p. 23-49, 2005.

MORAIS-TEIXEIRA, E.D. et al. The in vitro leishmanicidal activity of hexadecylphosphocholine (miltefosine) against four medically relevant Leishmania species of Brazil. Memórias do Instituto Oswaldo Cruz, Rio de Janeiro, v. 106, n. 4, p. 475-478, 2011.

MOSMANN, T. Rapid colorimetric assay for cellular growth and survival: application to proliferation and cytotoxicity assays. Journal of Immunological Methods, v. 65, n. 1-2, p. 55-63, 1983.

PAREKH, P. et al. Downregulation of cyclin D1 is associated with decreased levels of p38 MAP kinases, Akt/PKB and Pak1 during chemopreventive effects of resveratrol in liver cancer cells. Experimental and Toxicologic Pathology, v. 63, n. 1-2, p. 167-173, 2011.

PASSOS, C. L. A. et al. Leishmanicidal effect of synthetic trans-resveratrol analogs. PLoS One,San Francisco, v. 10, n. 10, p. e0141778, 2015.

RUGANI, J.N. et al. Intraspecies susceptibility of Leishmania (Viannia) braziliensis to antileishmanial drugs: Antimony resistance in human isolates from atypical lesions.

Biomedicine and Pharmacotherapy, v. 108, n. 2018, p. 1170-1180, 2018.

SILVEIRA, F.T. et al. Immunopathogenic competences of Leishmania (V.) braziliensis and L. (L.) amazonensis in American cutaneous leishmaniasis.Parasite Immunology, v. 31, n. 8, p. 423-431, 2009.

SOLOMON, V. R. et al. Synthesis and antimalarial activity of side chain modified 4aminoquinoline derivatives. Journal of Medicinal Chemistry, Washington,v. 50, n. 2, p. 394-398, 2007.

TUNG, B. T. et al. Anti-inflammatory effect of resveratrol in old mice liver. Experimental 
Gerontology, v. 64, p. 1-7, 2015.

ULIANA, S. R. B. et al. Chemotherapy of leishmaniasis: present challenges. Parasitology, Cambridge, v. 145, n. 5, p. 464-480, 2018.

WHO.Research priorities for Chagas disease, human African trypanosomiasis and leishmaniasis. WHO, Geneva, Switzerland, 2012. Disponível em:

http://apps.who.int/iris/bitstream/handle/10665/77472/WHO_TRS_975_eng.pdf;jsessionid= 72CF7AFCE69E55FD52DE89DCCD1A5C11?sequence=1. Acessoem: 23 nov. 2018.

YARDLEY, V. et al. The sensitivity of clinical isolates of Leishmania from Peru and Nepal to miltefosine. American Journal of Tropical Medicine and Hygiene, Cleveland, v. 73, n. 2, p. 272-275, 2005.

$\mathrm{XIA}, \mathrm{N}$. et al. Antioxidant effects of resveratrol in the cardiovascular system. British Journal of Pharmacology, Weinheim, v. 174, n. 12, p. 1633-1646, 2017. 